\title{
Upconversion assisted self-pulsing in a high-concentration erbium doped fiber laser
}

\author{
Sergey Sergeyev ${ }^{1}$, Kieran O’Mahoney ${ }^{1}$, Sergei Popov ${ }^{2}$, and Ari T. Friberg ${ }^{2,3,4}$ \\ ${ }^{1}$ Waterford Institute of Technology, Optics Research Group, Cork Road, Waterford, Ireland \\ Fax: + 35351 302679, emails: sergey.sergeyev@gmail.com, ssergeyev@wit.ie \\ ${ }^{2}$ Royal Institute of Technology (KTH), Department of Microelectronics and Applied Physics, \\ Electrum 229, SE-164 40 Kista, Sweden \\ ${ }^{3}$ Helsinki University of Technology, Department of Engineering Physics, FI-02015 TKK, Finland \\ ${ }^{4}$ University of Joensuu, Department of Physics and Mathematics, FI-80101 Joensuu, Finland
}

\begin{abstract}
We report results on experimental and theoretical characterisation of self-pulsing in high concentration erbium doped fibre laser which is free from erbium clusters. Unlike previous models of self-pulsing accounting for pair-induced quenching (PIQ) on the clustered erbium ions, new model has been developed with accounting for statistical nature of the excitation migration and upconversion and resonance-like pump-to-signal intensity noise transfer. The obtained results are in a good agreement with the experimental data.
\end{abstract}

PACS: 42.55.Wd, 42.55.Xi 42.65.Sf

Keywords: fibre lasers, upconversion, erbium, optical instabilities

\section{Introduction}

Self-pulsing in high concentration erbium doped fibre lasers (HC EDFLs) has been intensely studied during the last 15 years in the context of applications in communications, refelectometry, distributed fibre optic sensing, medicine etc. [1-9]. For a long time the presence of the clustered erbium ions and their behaviour as a saturable absorber has been considered as the only possible mechanism responsible for the self-pulsing [1-6, 9]. However, detailed microscopic study of erbium-doped glasses by means of X-ray absorption fine structure spectroscopy (XAFS) has found a short range coordination order of erbium ions rather than pair-clustering [10]. As follows from XAFS experiments, the pair-correlation function has maxima at $R_{1}=3.5 \AA$ and $R_{2}=3.9 \AA$, whereas it takes approximately a constant value for $R>4.5 \AA$ [10]. It was found also that suppression of the short-range order leads to improved characteristics of high concentration erbium doped fibre amplifiers and lasers and 
can be realized by increasing the solubility of erbium in host matrix (co-doping by $\mathrm{Al}$ [11] or using phosphate glass [12]) or by modification of deposition process (Direct Nanoparticle Deposition [13]). Application of such fibres as an active media for HC EDFLs resulted in auto-oscillations with two characteristic frequencies of about $10 \mathrm{kHz}$ and $100 \mathrm{kHz}$ with the pump power thresholds close and 10 times exceeding the first threshold correspondently [78]. Thus, unlike pair-clusters, alternative approaches to self-pulsing in HC EDFLs have to be developed. Although the model explaining mechanism of the auto-oscillations in HC EDFLs with accounting for upconversion processes is still absent, it was already experimentally shown in Ref. 8 that pump-to-signal noise transfer can play a significant role in appearance of low frequency self-pulsing. In addition, power-dependent thermo-induced lensing has been suggested as a mechanism for high-frequency (100 kHz) self-pulsing [7].

In this paper we develop an advanced model of HC EDFL accounting for the statistical nature of migration and upconversion processes [14-22]. We apply this model to calculate the pump-to-signal intensity noise transfer function and demonstrate that resonance behaviour of this function can lead to appearance of the low- and high-frequency auto-oscillations in the limited range of erbium concentrations. We show that the obtained results are in a good agreement with our experimental data.

\section{Experimental and theoretical characterisation of low-frequency self-pulsing in high concentration erbium doped fibre laser}

Experimental set-up is shown in Fig.1. High concentration $\left(\mathrm{C}_{\mathrm{Er}}=3.7 \times 10^{25}\right.$ ions $\left./ \mathrm{m}^{3}\right)$ erbium doped fibre (Liekki Er40-4/125) of 10 m length have been pumped trough WDM multiplexers by laser diodes with wavelengths of $978 \mathrm{~nm}$ and $1480 \mathrm{~nm}$ and the pump power slightly above the lasing threshold. The resulting auto-oscillations have been obtained without any pump modulation (Fig. 2). As follows from Fig. 2 (c), low frequency ( $\sim 3 \mathrm{kHz})$ and high frequency $(\sim 150 \mathrm{kHz})$ auto-oscillations are dominating.

For theoretical characterization of the low-frequency auto-oscillations we develop a new model of HC EDFL with taking into account statistical nature of migration-assisted upconversion [14-22] and pump-to-signal intensity noise transfer [5].

The derivation starts with the following rate equations which describes high concentration erbium doped fibre laser with accounting for excitation migration and upconversion and pump at $980 \mathrm{~nm}$ 


$$
\begin{aligned}
\frac{d n_{1 k}}{d t} & =\delta_{1} n_{2 k}+\left(1-n_{1 k} \beta_{s}-n_{2 k}\right) I_{L}-n_{1 k}-2 n_{1 k} \sum_{i=1, i \neq k}^{n_{1} N} P_{k i}-n_{1 k} \sum_{j=1, j \neq k}^{N} W_{k j}+\sum_{j=1, j \neq k}^{N} W_{k j} n_{1 j}, \\
\frac{d n_{2 k}}{d t} & =\left(1-n_{1 k}-n_{2 k}\right) I_{p}-\delta_{1} n_{2 k}+n_{1 k} \sum_{i=1, i \neq k}^{n_{1} N} P_{k i}, \\
\frac{d I_{L}}{d t} & =\delta_{2} I_{L}\left[\alpha_{L} L\left(\beta_{L} n_{1}+n_{2}-1\right)-k_{L}\right]
\end{aligned}
$$

Here time $t$ is normalized to lifetime $\tau_{1}$ of first excited level; $\delta_{1}=\tau_{1} / \tau_{2}, \delta_{2}=\tau_{1} / \tau_{r}$, where $\tau_{2}, \tau_{r}$ are the lifetime of the first excited level and photon intracavity round-trip time; $n_{1 k}, n_{2 k}$ are the probabilities of the localization of the excitation on ion number $k$ located at the first and the second excited level correspondently, $N$ is the total number of ions, and $n_{1}, n_{2}$ are the populations of the first and the second excited levels $\left(n_{i}=\lim _{N \rightarrow \infty} \sum_{k=1}^{N} n_{i k} / N\right)$. Further, $\beta_{L}=\left(\sigma_{a L}+\sigma_{e L}\right) / \sigma_{a L}$; pump $I_{p}$ and lasing power $I_{L}$ are normalized on the corresponding saturation powers: $I_{i s}=h c A c_{E r}\left(\lambda_{i} \alpha_{i} \tau\right), \quad(i=s, p)$; $A$ is an effective area of the erbium distribution, $\alpha_{i}=\Gamma \sigma_{a i} C_{E r}$ is the small signal absorption of HC EDFL at wavelength $\lambda_{i} ; \sigma_{a i}$ and $\sigma_{e i}$ are the absorption and emission cross-sections at the signal $(i=s)$ and the pump $(i=p)$ wavelength, $\Gamma_{i}=1-\exp \left(-2 b^{2} / w_{i}^{2}\right)$ is overlap factor, $b$ is the erbium ion dopant radius, $w_{i}$ is mode field radius at wavelength $\lambda_{i}$; $L$ is fibre length, $c_{E r}$ is the concentration of $\mathrm{Er}^{3+}$ ions. The rates of upconversion $P_{k i}$ and migration $W_{k j}$ (from ion $k$ to ions $i$ and $j$ ) for the dipoledipole mechanism of excitation energy transfer are given as [14-22]

$$
P_{k i}=\left(\frac{R_{u p}}{R_{k i}}\right)^{6}, W_{k j}=\left(\frac{R_{m}}{R_{k j}}\right)^{6},
$$

where $R_{u p}$ and $R_{m}$ are the critical distances for upconversion and migration respectively [8]. It is easy to show that the excited state absorption (ESA) in Eq. 1 can be neglected if inequality $I_{p}\left(\sigma_{\text {esa }}+\sigma_{a p}\right) / \sigma_{a p} \ll<\delta_{1}$ holds. This is justified because of we consider low pump powers of 1.03 and just 10.03 times of the first threshold pump power.

As follows from Eqs.1, the model, unlike erbium cluster model, takes into account only variance in the distances between excited erbium ions and variance in the interaction probabilities, correspondingly. Averaging over the variance in the separations with taking into 
account cw operation $\frac{d n_{i k}}{d t}=0, \frac{d I_{L}}{d t}=0$ and mean-filed approximation $\sum_{j=1, j \neq k}^{N} W_{k j} n_{1 j} \approx n_{1} \sum_{j=1, j \neq k}^{N} W_{k j}$ [14-22], we derive the following system of macroscopic equations from which the excited levels populations $n_{1}, n_{2}$, cw rate of upconversion $W_{\text {up }}$ and the lasing power $I_{L}$ can be found as function of the normalised pump power $I_{p}$ and normalised concentration of erbium ions $\gamma=c_{E r} / c_{u p}\left(c_{u p}=\left(\frac{4 \pi}{3} R_{u p}^{3}\right)^{-1}\right.$ is the critical concentration for upconversion $)$.

$$
\begin{aligned}
& n_{1}=\frac{B(1+B)^{-1}\left(\sqrt{A} n_{1}+\sqrt{r / 2}\right) F\left(\frac{\sqrt{\pi} \gamma\left(\sqrt{A} n_{1}+\sqrt{r / 2}\right)}{2 \sqrt{1+B}}\right)}{\sqrt{A} n_{1}+\sqrt{r / 2} F\left(\frac{\sqrt{\pi} \gamma\left(\sqrt{A} n_{1}+\sqrt{r / 2}\right)}{2 \sqrt{1+B}}\right)}, \\
& n_{2}=\frac{2\left(1-n_{1}\right) I_{p}+\left(1-\beta_{L} n_{1}\right) I_{L}-n_{1}}{\delta_{1}+2 I_{p}+I_{L}}, \\
& W_{u p}=\frac{\left(1-n_{1}-n_{2}\right) I_{p}+\left(1-\beta_{L} n_{1}-n_{2}\right) I_{L}-n_{1}}{n_{1}}, \\
& \beta_{L} n_{1}-n_{2}-1=\frac{k_{L}}{\alpha_{L} L} .
\end{aligned}
$$

Here $A=2-\frac{\delta_{1}-I_{L}}{\delta_{1}+I_{p}}, B=\beta_{L} I_{L}+\frac{\delta_{1}-I_{L}}{\delta_{1}+I_{p}} I_{p}, F(x)=1-\sqrt{\pi} x \exp \left(x^{2}\right) \operatorname{erfc}(x), \quad r=\left(R_{m} / R_{u p}\right)^{6}$.

Close to the cw operation the dynamic behaviour of HC EDFL can be caused by small pump power fluctuations, i.e. we can write $I_{p}(t)=I_{p}+\Delta I_{p}(t), n_{i k}(t)=n_{i k}+x_{i k}(t)(i=1,2), I_{L}(t)=I_{L}+x_{3}(t)$. By substituting these expressions into Eqs. (1) with accounting averaging procedure, we result in the following equations

$$
\begin{aligned}
\frac{d x_{1}}{d t} & =-a_{1}\left(\gamma, I_{p}\right) x_{1}+a_{2}\left(\gamma, I_{p}\right) x_{2}-a_{3} x_{3}, \\
\frac{d x_{2}}{d t} & =b_{1}\left(\gamma, I_{p}\right) x_{1}-b_{2}\left(I_{p}\right) x_{2}+b_{3}\left(\gamma, I_{p}\right) \Delta I_{p}, \\
\frac{d x_{3}}{d t} & =c_{1}\left(\gamma, I_{p}\right) \beta_{L} x_{1}+c_{1}\left(\gamma, I_{p}\right) x_{2} .
\end{aligned}
$$

Where 


$$
\begin{aligned}
& a_{1}\left(\gamma, I_{p}\right)=1+2 W_{u p}\left(\gamma, I_{p}\right)+\beta_{L} I_{L}\left(\gamma, I_{p}\right), a_{2}\left(\gamma, I_{p}\right)=\delta_{1}-I_{L}\left(\gamma, I_{p}\right), \quad a_{3}=\frac{k_{L}}{\alpha_{L} L}, \\
& b_{1}\left(\gamma, I_{p}\right)=W_{u p}\left(\gamma, I_{p}\right)-I_{p}, \quad b_{2}\left(I_{p}\right)=\delta_{1}-I_{p}, \quad b_{3}\left(\gamma, I_{p}\right)=1-n_{1}\left(\gamma, I_{p}\right)-n_{2}\left(\gamma, I_{p}\right), \\
& c_{1}\left(\gamma, I_{p}\right)=\delta_{2} L \alpha_{L} I_{L}\left(\gamma, I_{p}\right)
\end{aligned}
$$

Here $x_{1(2)}=\left\langle x_{1(2) k}\right\rangle_{s_{1}, s_{2}}$ are fluctuations of the populations of the first and second excited levels averaged over the stochastic variables $S_{1}$ and $S_{2}$ which are presenting the excitation upconversion and migration processes [14, 22]. During the averaging procedure, the approximation

$$
\left\langle S_{1} x_{1 k}\right\rangle /\left\langle x_{1 k}\right\rangle \approx W_{u p}
$$

has been used. Taking the Laplace transform of the Eqs. (4) : $\tilde{x}_{i}(p)=\int_{0}^{\infty} \exp (-p t) x_{1}(t) d t$ and defining $p=-i \omega$, we can obtain the following normalized transfer function for the pump-tosignal noise transfer which describes, in the frequency domain, the response of laser power to the perturbations in the pump rate [5]:

$$
H_{p}\left(\omega, I_{p}, \gamma\right)=\frac{x_{3} / I_{L}^{(c w)}}{\Delta I_{p} / I_{p}^{(c w)}},
$$

As follows from Ref. 5, relative intensity noise (RIN) for the HC EDFL is proportional to $\left|H_{p}\left(\omega, I_{p}, \gamma\right)\right|^{2}$. As a result, we find the $\left|H_{p}(\omega)\right|^{2}$ from Eqs. 4 and 5 as following:

$$
\begin{aligned}
& \left|H_{p}(\omega)\right|^{2}=\left(c_{1}\left(0.1, I_{p}\right) \frac{\gamma}{0.1}\right)^{2} b_{3}\left(\gamma, I_{p}\right)^{2} I_{p}^{2}\left[\left(a_{1}\left(\gamma, I_{p}\right)+\beta_{L} a_{2}\left(\gamma, I_{p}\right)\right)^{2}+\omega^{2}\right]^{2} \times \\
& I_{L}\left(\gamma, I_{p}\right)^{-2}\left\{\begin{array}{l}
{\left[\omega^{2}\left(a_{1}\left(\gamma, I_{p}\right)+b_{2}\left(I_{p}\right)\right)^{2}-c_{1}\left(0.1, I_{p}\right) \frac{\gamma}{0.1} a_{3}\left(b_{1}\left(\gamma, I_{p}\right)+\beta_{L} b_{2}\left(I_{p}\right)\right)\right]^{2}+} \\
\left.+\omega^{2}\left[-a_{1}\left(\gamma, I_{p}\right) b_{2}\left(I_{p}\right)+\omega^{2}+b_{1}\left(\gamma, I_{p}\right) a_{2}\left(\gamma, I_{p}\right)-c_{1}\left(0.1, I_{p}\right) \frac{\gamma}{0.1} \beta_{L} a_{3}\right]^{2}\right\}^{-1}
\end{array}\right.
\end{aligned}
$$

Here coefficients $a_{i}, b_{i}$ and $c_{1}$ have been calculated with help of Eqs. (5). 


\section{Results and discussion}

Pump-to-signal transfer function $\left|H_{p}\left(\omega, I_{p}, \gamma\right)\right|^{2}$ as a function of frequency, pump power and normalised concentration has been calculated for the following parameters: $k_{L} /\left(\alpha_{L} L\right)=0.2$, $r=60, \gamma=0.1,0.33,0.5 ; \alpha_{L} L \delta_{2}=10^{6}, \delta_{1}=10^{3}$. The results of calculations are shown in Fig.3 for the pump power $I_{p}=1.03 I_{p, t h}(\mathrm{a})$ and $I_{p}=10.03 I_{p, t h}(\mathrm{~b})\left(I_{p, t h}\right.$ is the threshold pump power $)$. The conditions of the constant number of ions, i.e. $N \sim \alpha_{L} L=$ const, has also been used. As follows from Fig.3 (a, b), when the pump power close $\left(I_{p}=1.03 I_{p, t h}\right)$ and 10 times exceed the first threshold value $\left(I_{p}=10.03 I_{p, t h}\right)$ function $\left|H_{p}\left(\omega, I_{p}, \gamma\right)\right|^{2}$ demonstrates resonance-like behaviour at the low- $(\sim 10 \mathrm{kHz})$ and high-frequencies $(\sim 100 \mathrm{kHz})$. This leads to increased lasing intensity noise which works as an external resonance force and, therefore, low- and highfrequency frequency self-pulsing appears ([8] and Fig.2 (a-c)). As folows from Eqs. 8, $\left|H_{p}\left(\omega, I_{p}, \gamma\right)\right| \sim \alpha_{L} \delta_{2} \sim c_{E r}$ in the maximum and, therefore, the function increase with increased concentration of erbium ions (Fig3 (a, b)). Resonance frequency for low-frequency autooscillations coincides with frequency of relaxation oscillations $\omega_{R}$ which can be written as follows: $\omega_{R}=\sqrt{k_{L}\left(I_{p} / I_{p t h}-1\right) /\left(\tau_{1}(\gamma) \tau_{r}\right)}$, where $\tau_{1}(\gamma)$ is the lifetime of the first excited level with accounting for upconversion [23]. Upconversion processes result in the decreased lifetime and, therefore, in increased resonance frequency (Fig. 3 (a)).

In conclusion, we have presented a new advanced model of HC EDFL accounting for the statistical nature of migration and upconversion processes. By calculating the pump-to-signal intensity noise transfer function, we have demonstrated that resonance behaviour of this function can lead to appearance of the low- and high-frequency auto-oscillations in the limited range of erbium concentrations. We show that the obtained results are in a good agreement with our experimental data and the data obtained in Ref. 8.

S. Sergeyev acknowledges financial support from the Enterprise Ireland Commercialization fund, and S. Popov and A.T. Friberg acknowledge funding from the Swedish Foundation for Strategic Research (SSF). A.T. Friberg also thanks the Academy of Finland for funding under the Finland Distinguished Professor program.

\section{References}

[1] M.J.F. Digonnet, Ed., Rare-Earth Doped Fiber Lasers and Amplifiers (Marcel Dekker, New York, 2001) 
[2] P.L. Boudec, P.L. Francois, E. Delevaque, J.F. Bayon, F. Sanchez, G. Stephan, Opt. Quantum Electron. 25, 501 (1993)

[3] F. Sanchez, M.L. Flohic, G. Stephan, P.L. Boudec, P.L. Francois, IEEE J. Quantum Electron. 31, 481 (1995)

[4] J. Daniel, J.-M. Costa, P. LeBoudec, G. Stephan, E. Sanchez, JOSA B 15 (1998)

[5] H.L. An, E.Y.B. Pun, X.Z. Lin, H.D. Liu, IEEE Photon. Technol. Lett. 11, 803 (1999)

[6] P. Besnard, F.Ginovart, P. Le Boudec, F. Sanchez; G.M. Stephan, Opt. Communications, 205, 187 (2002).

[7] A.V. Kir’yanov, N.N. Il’ichev, Yu.O. Barmenkov, Laser Phys. Lett. 1, 194 (2004)

[8] Yu.O. Barmenkov, A.V. Kir’yanov, Optics Express, 12, 3171 (2004)

[9] A. Bahrampour, S. Keyvaninia; M. Karvar, Optical Fiber Technol. 14, 54 (2008)

[10] P. M. Peters and S. N. Houde-Walter, J. Non-Cryst. Solids 328,162 (1998).

[11] J. Lægsgaard, Phys. Rev. B, 65174114 (2002).

[12] B.-C. Hwang, S. Jiang, T. Luo, K. Seneschal, G. Sorbello, M. Morell, F. Smektala, S. Honkanen, J. Lucas, N. Peyghambarian, IEEE Photon. Technol. Lett. 13, 197 (2001).

[13] S.Tammela, M. Hotoleanu, P. Kiiveri, H. Valkonen, S. Sarkilahti, K. Janka, in Conference on Optical Fiber Communications, Vol. 1, 2003 OSA Technical Digest Series (OSA, Washingtaon, D.C., 2003), pp. 376-377.

[14] S. Sergeyev, S. Popov, JEOS - Rapid publications , 207027 (2007)

[15] S. Sergeyev, S. Popov, A. T. Friberg, JOSA B 231540 (2006)

[16] S. Sergeyev, S. Popov, A. T. Friberg, Optics Lett. 301258 (2005)

[17] D. Khoptyar, S.Sergeyev, B. Jaskorzynska, IEEE J. Quant. Electron., 41205 (2005)

[18] D. Khoptyar, S.Sergeyev, B. Jaskorzynska, JOSA B, 22582 (2004)

[19] S.Sergeyev, Electronics Lett., 39511 (2003)

[20] S. Sergeyev, D. Khoptyar, B. Jaskorzynska, Physical Rev. B, 652331 (2002)

[21] S.V.Sergeyev, B.Jaskorzynska, Phys.Rev. B, 6215628 (2000).

[22] S. Sergeyev, D. Khoptyar, in Laser Optics 2006: Solid State Lasers and Nonlinear Frequency Conversion, edited by Vladimir I. Ustyugov, Proc. of SPIE Vol. 6610, 66100L (2007) •

[23] YA I. Khanin, Fundamentals of Laser Dynamics (Cambridge Int Science Publishing, 2005) 
Figure captions

Fig.1 High concentration erbium doped fibre laser

Fig. 2 Low- and high-frequency auto-oscillations in high concentration erbium doped fibre laser (a, b), high-frequency auto-oscillations (b), Fourier transform of the dynamics is shown in Fig. 2 (c).

Fig.3 Pump-to-signal intensity noise transfer function as function of frequency and normalised concentration of erbium ions: $\gamma=0.1$ (solid line), $\gamma=0.33$ (dotted line), $\gamma=0.5$ (dashed line). Pump power $I_{p}=1.03 I_{p, t h}$.(a), Pump power $I_{p}=10.03 I_{p, t h}$. 


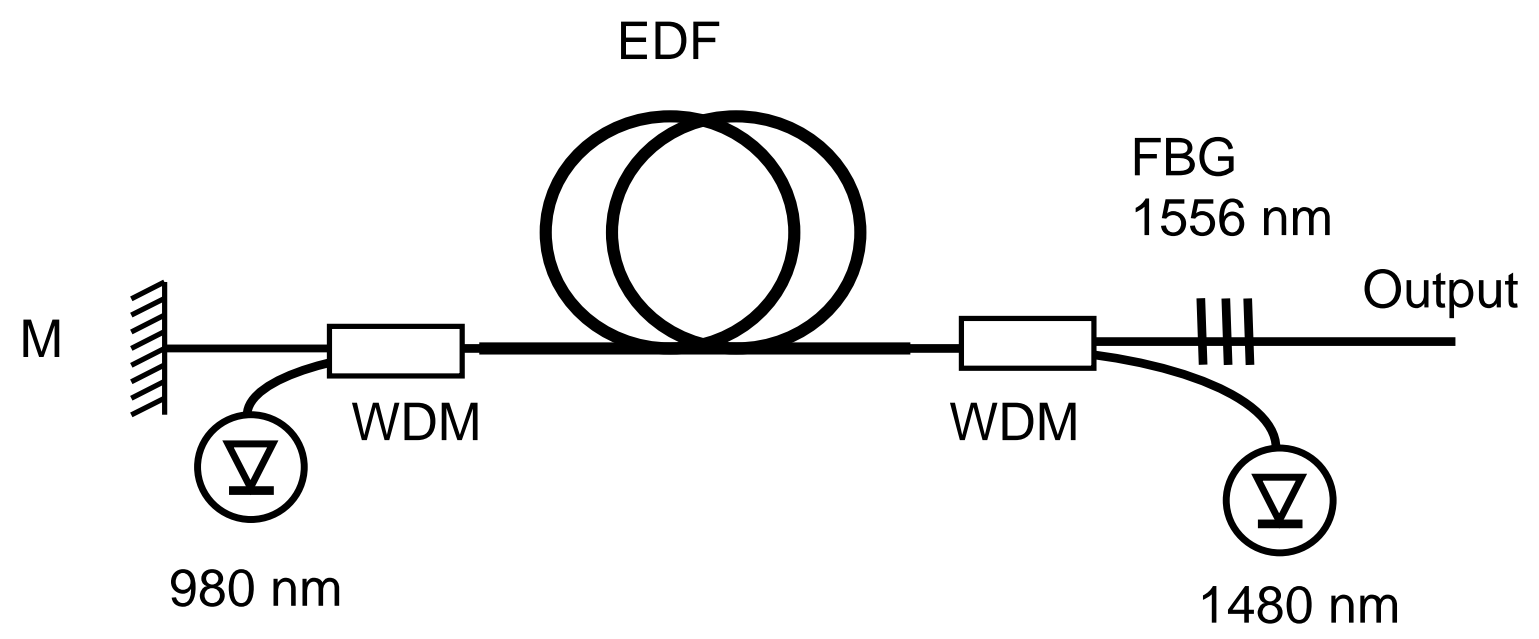

Fig.1 


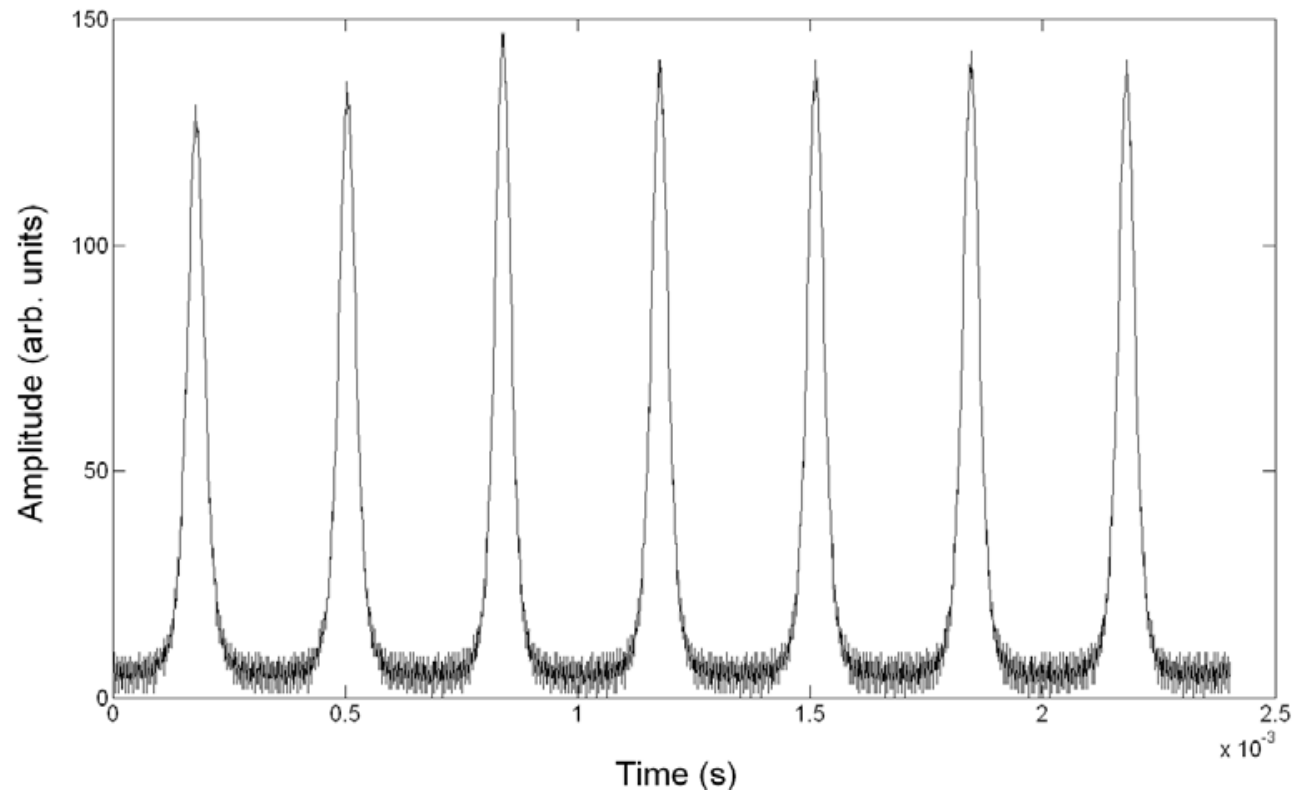

Fig.2 (a) 


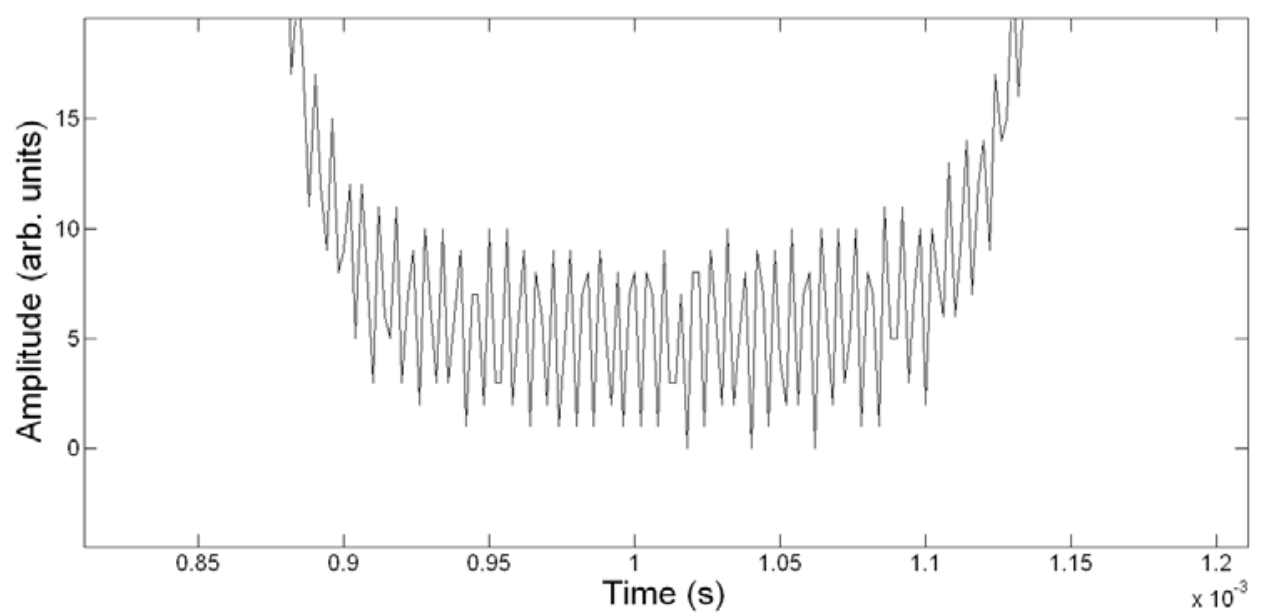

Fig. 2 (b) 


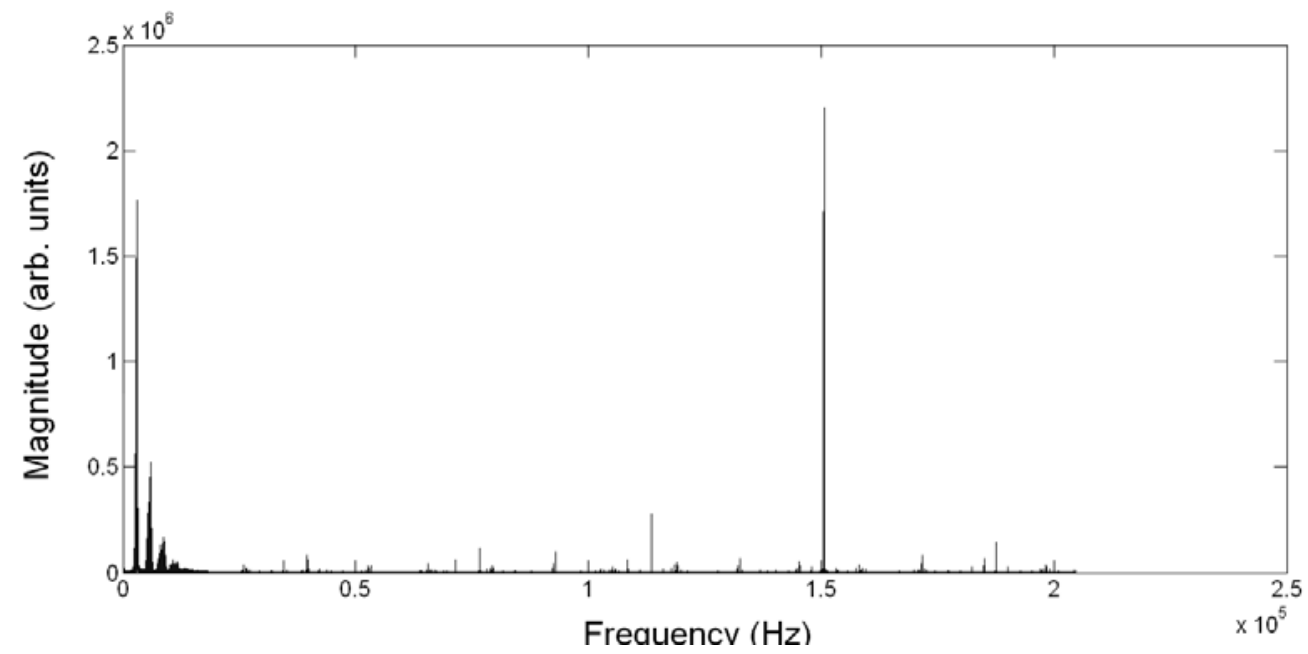

Fig. 2 (c) 


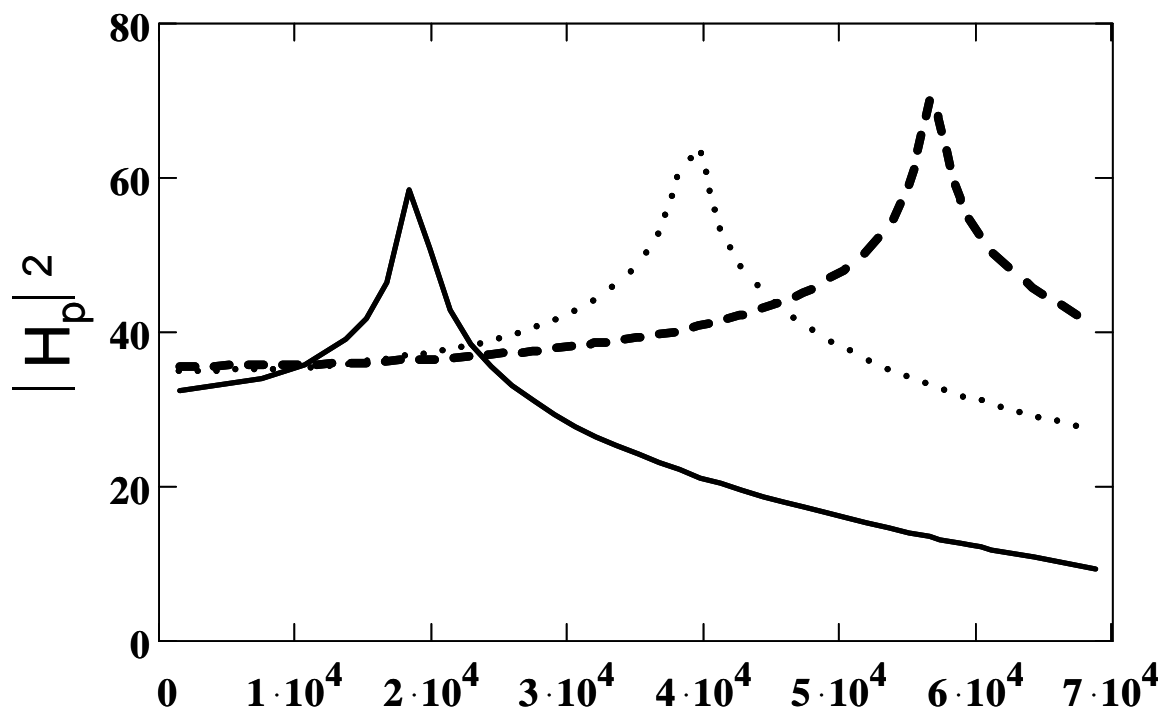

Frequency, $\omega(\mathbf{H z})$

Fig. 3 (a) 


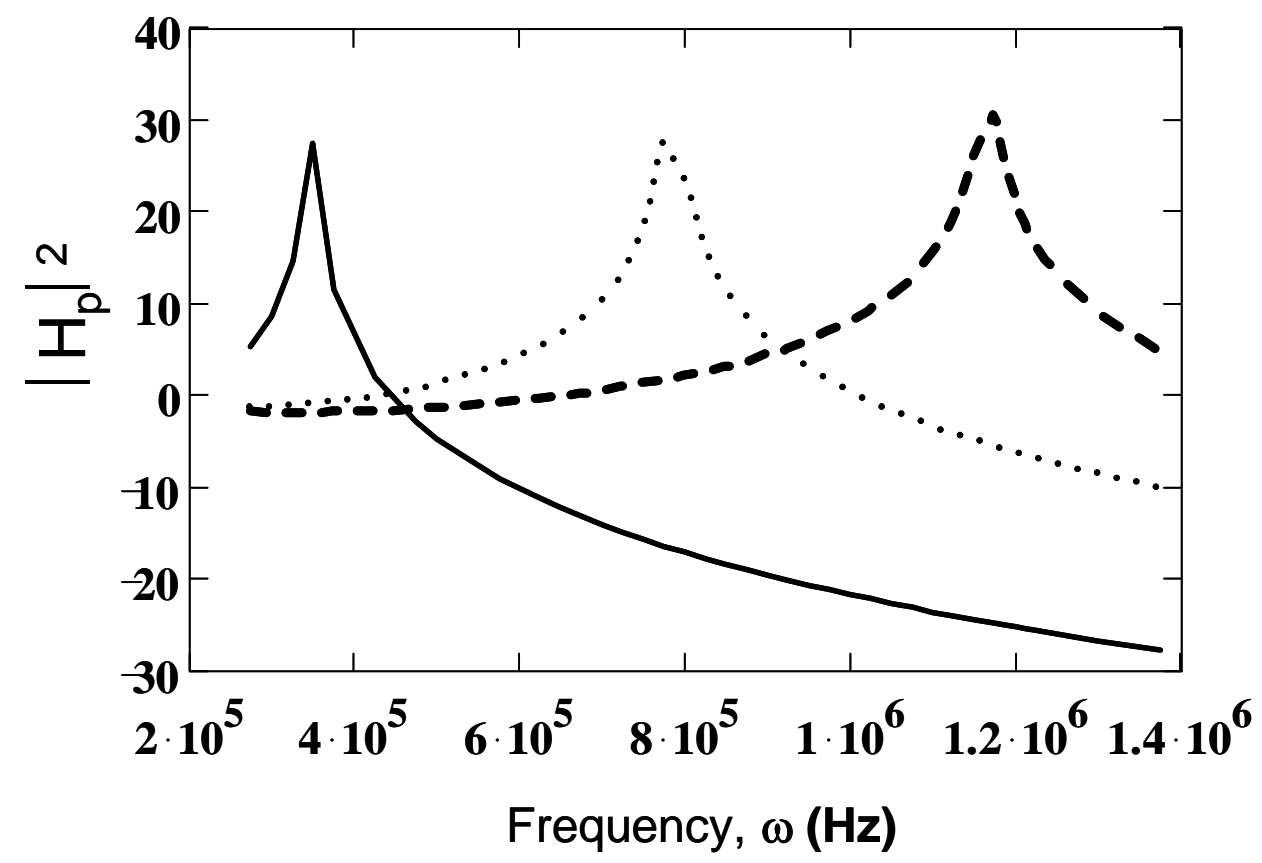

Fig.3 (b)

Fig.3 (b) 